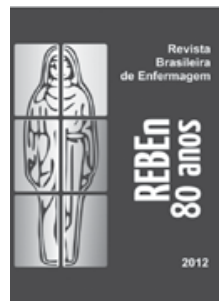

\title{
Transformações necessárias para o avanço da Enfermagem como ciência do cuidar
}

\author{
Necessary changes for advancing Nursing as caring science \\ Cambios necesarios para el desarrollo de la Enfermería como ciencia del cuidado
}

\section{Denise Elvira Pires de Pires ${ }^{1}$}

' Universidade Federal de Santa Catarina, Centro de Ciências da Saúde, Departamento de Enfermagem, Programa de PósGraduação em Enfermagem. Florianópolis-SC, Brasil.

\author{
Submissão: 02-09-2013 Aprovação: 03-09-2013
}

\section{RESUMO}

Objetivou-se refletir acerca dos desafios para fortalecer a Enfermagem enquanto ciência do cuidar. Sustenta-se na teoria sociológica articulando três abordagens: do materialismo histórico-dialético sobre o processo de trabalho em saúde e enfermagem; da sociologia das profissões em uma perspectiva crítica; e da filosofia da ciência. A argumentação está organizada considerando aspectos da Enfermagem enquanto disciplina, trabalho e profissão da saúde. Sustenta que a produção do conhecimento deve ser orientada pela finalidade do trabalho de cuidar de seres humanos com carências de saúde e pela defesa das condições necessárias para um fazer seguro e responsável. Conclui que, para fortalecer a Enfermagem, é necessário produzir conhecimentos que fundamentem as ações de cuidado, assim como as lutas políticas em defesa da profissão, do direito universal à saúde e a cuidados seguros e de qualidade.

Descritores: Enfermagem; Profissão; Cuidado; Ciência.

\section{ABSTRACT}

The article aimed to reflect upon the challenges involved in strengthening Nursing as a caring science. It is founded on the sociological theory, connecting three approaches: the historical-dialectic materialism perspective about the working process in health care and nursing; the sociology of professions from a critical perspective; and the philosophy of science. The discussion is organized considering the aspects of Nursing as a discipline, work and health care profession. It sustains that knowledge production should be driven both by the purpose of Nursing work which is providing care to human beings with health needs and to advocate for the indispensable work conditions to a safe and responsible practice. It concludes that to strengthening Nursing it is necessary to produce knowledge to support nursing care and the political actions defending safe work conditions, the universal right to health as well safe and high quality care.

Key words: Nursing; Profession; Care; Science.

\section{RESUMEN}

El artículo objetivó reflexionar sobre los desafíos para el fortalecimiento de la Enfermería como ciencia del cuidado. Se sustenta en la teoría sociológica articulando tres abordajes: del materialismo histórico-dialéctico sobre el proceso de trabajo en salud y Enfermería; de la sociología de las profesiones en una perspectiva crítica; y de la filosofía de la ciencia. La argumentación se organiza teniendo en cuenta aspectos de la Enfermería como disciplina, trabajo y profesión de salud. Se sustenta que la producción del conocimiento debe guiarse por la finalidad del trabajo de cuidar de seres humanos con carencias de salud y por la defensa de condiciones necesarias para un hacer seguro y responsable. Se concluye que para fortalecer la Enfermería es necesario producir conocimientos que fundamenten las acciones del cuidado, así como las luchas políticas en defensa de la profesión, del derecho universal a la salud y a los cuidados seguros y de calidad.

Palabras clave: Enfermería; Profesión; Cuidado; Ciencia. 


\section{INTRODUÇÃO}

Este artigo foi construído a partir de uma solicitação da Associação Brasileira de Enfermagem para abordá-lo na Conferência de Encerramento do $17^{\circ}$ Seminário Nacional de Pesquisa em Enfermagem - SENPE, ocorrido em Natal, no Rio Grande do Norte, em junho de 2013. Este Seminário tem o importante papel de propiciar reflexões sobre um dos pilares de uma profissão que é a produção de conhecimentos que sustentam o seu fazer.

A programação do $17^{\circ}$ SENPE foi bastante significativa, iniciando pelo tema central ao reconhecer a importância e os desafios que envolvem o "clássico e o emergente na pesquisa em Enfermagem". No temário verificou-se, também, o reconhecimento da importância da ética no processo de pesquisar, na realização do trabalho da Enfermagem e na formação de novos profissionais, além da importância de analisar criticamente o cenário no qual é realizado o trabalho de pesquisa, de prestação de cuidados e de formação de novos profissionais.

Na estrutura do temário identificou-se que a profissão, expressa na proposta da ABEn para o SENPE, reconhece a necessidade de discutir o "como pesquisar" incluindo metodologias e técnicas de pesquisa, assim como de propor temas político-profissionais pertinentes à finalidade do seu trabalho. Finalidade esta que responde ao critério mais importante para a caracterização de um trabalho como profissão - a sua utilidade social. É pelo fazer do conjunto dos profissionais de Enfermagem que a sociedade reconhece a utilidade deste trabalho, assim como a necessidade do mesmo ser organizado em padrões profissionais. Enquanto um trabalho do tipo profissional e disciplina científica do campo da saúde, a Enfermagem apresenta desafios permanentes. Desafios demandados pela dinâmica do cenário macro social no qual este trabalho se realiza, por mudanças no perfil demográfico e epidemiológico da população e pela complexidade inerente ao processo de cuidar de seres humanos com carências de saúde.

O conjunto do temário do seminário demonstrou o claro reconhecimento de que, para atuar com competência e segurança, é necessário fortalecer os conhecimentos já consolidados pela Enfermagem enquanto disciplina científica e, ao mesmo tempo, manter uma postura permanente de abertura ao novo. É preciso enfrentar os desafios sempre presentes em um trabalho que tem como identidade o cuidado a seres humanos com necessidades do campo da saúde. Um trabalho complexo e em permanente mudança, o que demanda da profissão sólida consistência científica e respeito a padrões éticos que reafirmem valores como a vida humana, a justiça social e o direito à saúde. É preciso, ainda, ter abertura para analisar criticamente os diferentes cenários, propostas terapêuticas e políticas de saúde.

O trabalho da Enfermagem desenvolve-se, majoritariamente, como um trabalho do tipo coletivo e em colaboração com outros profissionais e trabalhadores do campo da saúde. Fundamenta-se em um saber consolidado que deve fornecer bases para o agir cotidiano, além de subsidiar a proposição de caminhos para o enfrentamento de novos e velhos problemas do campo da saúde e específicos ao seu núcleo profissional. Os desafios são constantes para um trabalho que envolve seres humanos que vivem em sociedades históricas, em especial no caso da sociedade atual que é dialeticamente local e globalizada, influenciando tanto o surgimento e a disseminação de doenças quanto o acesso às informações sobre possibilidades terapêuticas.

Neste cenário, o presente artigo tem por objetivo refletir acerca dos desafios com vistas a fortalecer a Enfermagem enquanto ciência do cuidar. Foi organizado articulando aspectos da Enfermagem enquanto disciplina, trabalho e profissão da saúde e sustentado pela teoria sociológica. O referencial teórico articula formulações acerca do processo de trabalho em saúde e enfermagem com base no materialismo histórico-dialético, a sociologia das profissões em uma perspectiva crítica e a filosofia da ciência.

\section{A ENFERMAGEM E OS DESAFIOS PROFISSIONAIS}

O debate acerca da classificação de um trabalho como ocupação ou profissão é significativo e fundamenta-se nas características dos diferentes trabalhos e na capacidade de seus exercentes demonstrarem a importância de seu trabalho e a necessidade de disporem de prerrogativas legais de proteção. Dentre os atributos de profissão destaca-se: realizar um trabaIho que tenha utilidade social, ou seja, a sociedade reconhece a sua necessidade e importância; fundamentar-se em um saber especial a ser produzido pelos exercentes da profissão e transmitido pelos pares na formação de novos profissionais; dispor de autonomia para decidir sobre o seu trabalho; contar com legislação especifica que define quem pode exercer a profissão; dispor de um código de ética que estabeleça padrões orientadores para o agir profissional; e contar com entidades que representem a profissão na sociedade e que defendam os parâmetros ético-legais e as condições requeridas para este fazer.

A base teórica destes atributos origina-se de Max Weber ${ }^{(1)}$ e da Sociologia das Profissões, com contribuição especial da produção Norte Americana das décadas de 1970 e $1980^{(2-5)}$ e utilizada internacionalmente ${ }^{(6)}$. No Brasil, tem sido utilizada em estudos sobre o trabalho em saúde destacando-se Machado $^{(7)}$, Pereira e Pereira Neto ${ }^{(8)}$ e, em uma perspectiva crítica, por Pires ${ }^{(9-11)}$ e Bellaguarda e colaboradores ${ }^{(12)}$.

O debate sobre ocupação e profissão não diz respeito somente a questões teóricas, envolve a dinâmica da vida em sociedade e a luta política que demanda analisar os atributos da sociologia das profissões relativizando sua aplicação. Ou melhor, ao colocar atributos teóricos em práticas concretas podemos questionar o tipo ideal formulado. Assim, analisar o trabalho da Enfermagem relacionado aos atributos de profissão requer olhar para a utilidade social deste trabalho, assim como para os determinantes histórico-sociais de seu papel no campo multiprofissional de saúde. Diferentes posições e interesses se expressam no aparelho de estado, no campo jurídico e no legislativo em permanente movimento. Os resultados refletem a correlação de forças dos diferentes grupos 
de trabalhadores que lutam por reconhecimento social, e do movimento de empregadores que motivados pela dimensão econômica atuam, na maioria das vezes, fortalecendo a estratificação e defendendo a atribuição de diferentes valorizações econômicas e de status a distintos trabalhos. No campo da saúde o modelo da biomedicina e a lógica da organização capitalista do trabalho resultaram em fragmentação dos seres humanos e em fragmentação e hierarquização do trabalho. Em sentido oposto, os conceitos de integralidade e interdisciplinaridade tratam da necessidade de redirecionar a prática assistencial para o foco no ser humano e nas trocas entre os diversos saberes com vistas a impactar positivamente a qualidade, a segurança e a satisfação de usuários e profissionais. Os resultados de práticas colaborativas interdisciplinares poderiam contribuir, ainda, para a redução dos crescentes custos do setor e para a redução dos efeitos adversos causados pelo desperdício e pelos danos ambientais.

Neste cenário encontra-se quem defenda que a Enfermagem é uma semiprofissão por não responder ao "tipo ideal" descrito para profissões como medicina, advocacia, engenharia, clérigos e professores universitários ${ }^{(7)}$. No entanto, considerando-se a mesma base teórica que caracteriza determinados grupos de trabalhadores como profissão e outros como ocupação ou "semiprofissão", podemos afirmar que a Enfermagem é uma profissão do campo da saúde,

reconhecida desde a segunda metade do século que XIX quando Florence Nightingale acrescenta atributos a um campo de atividades de cuidado à saúde desenvolvidas, milenarmente, por indivíduos ou grupos com diferentes qualificações e em diferentes cenários. Com Florence, o cuidado ganha especificidade no conjunto da divisão do trabalho social, é reconhecido como um campo de atividades especializadas e necessárias/úteis para a sociedade e que, para o seu exercício, requer uma formação especial e a produção de conhecimentos que fundamentem o agir profissional(11).

Considerando-se ainda os atributos de profissão aplicados à realidade atual da Enfermagem brasileira, podemos afirmar que se trata de uma profissão por: a) ser desenvolvida por trabalhadores qualificados e especializados para a realização de uma atividade socialmente necessária, fundamental para a vida humana - o cuidado de pessoas com necessidades de atenção/assistência em saúde; b) contar com entidades que a representam no conjunto da sociedade formulando e propondo regras para o exercício profissional, destacando-se a definição de um código de ética que orienta o agir profissional em bases moralmente aceitáveis; c) ser reconhecida pela sociedade e pelo Estado, ao contar com uma Lei que define quem pode exercer a profissão e os parâmetros para o seu exercício; e, d) dominar "um campo de conhecimentos que lhe dá competência para cuidar das pessoas, em todo o seu processo de viver", no âmbito individual e coletivo, e entendendo que este processo de cuidar tem três dimensões básicas "cuidar de indivíduos e grupos, da concepção à morte"; o educar/pesquisar "que envolve o educar intrínseco ao processo de cuidar, a educação permanente no trabalho, a formação de novos profissionais e a produção de conhecimentos que subsidiem o processo de cuidar"; e a "dimensão administrativo-gerencial de coordenação do trabalho coletivo da enfermagem, de administração do espaço assistencial, de gerenciamento do cuidado e de participação no gerenciamento da assistência de saúde e institucional"(11).

Ao pensar a Enfermagem como trabalho e profissão, é apropriada a ideia de fortalecer/reafirmar conquistas e ser capaz de captar o emergente/tendências. Em cenários complexos, de incertezas e em constante mudança cabe usar como referência a defesa do direito à vida, à saúde e a cuidados seguros e de qualidade. Assim como, defender a valorização da profissão orientando-se pelo agir ético e pelo exercício do pensamento crítico para análise das políticas de saúde, de seu saber, de sua prática e do paradigma hegemônico de ciência, considerando os cenários sócio econômico, epidemiológico e demográfico da população. Enquanto trabalho e profissão é fundamental, ainda, produzir conhecimentos acerca de padrões éticos e do perfil da força de trabalho (em termos de qualidade e quantidade) requerida para um agir profissional seguro para profissionais e usuários dos serviços de saúde. É necessário produzir conhecimentos que instrumentalizem o processo de tomada de decisões e a luta da categoria por condições adequadas de trabalho, aqui entendidas em sentido ampliado, o que, como formulado por Pires, Lorenzetti e Gelbcke ${ }^{(13)}$, inclui: salário; jornada; regras de aposentadoria; ambiente de trabalho adequado; instrumentos de trabaIho seguros, tecnologicamente apropriados e em quantidade adequada; equipamentos de proteção individual e coletiva; situação contratual; número e qualificação da força de trabaIho; divisão do trabalho. Produzir conhecimentos que fundamentem as lutas por legislação trabalhista apropriada para o trabalho na saúde e na Enfermagem e por políticas públicas adequadas às necessidades de saúde da população em cada momento histórico. E produzir conhecimentos sobre gestão em saúde e Enfermagem, diferentes sistemas, culturas e práticas de saúde e sobre protocolos clínicos e intervenções terapêuticas.

Resgatando as ideias de núcleo e campo formuladas por Campos $^{(14)}$, produzir conhecimentos que fundamentem o núcleo profissional "Enfermagem" para sua atuação qualificada no campo da saúde atuando em defesa da vida, do direito à saúde e do viver com qualidade e segurança.

Cabe aos profissionais de Enfermagem produzir conhecimentos sobre o que identifica o trabalho da Enfermagem no trabalho coletivo multiprofissional em saúde; o que significa um trabalho interdisciplinar e como a Enfermagem se coloca neste debate; qual a contribuição da Enfermagem para a assistência de saúde incluindo a promoção da saúde, práticas de cuidado, o viver com incapacidades e limitações e cuidados no processo de morrer.

Produzir conhecimentos sobre ética, bioética e suas implicações no fazer da Enfermagem. Produzir conhecimentos sobre história do campo da saúde e da Enfermagem e sobre educação em saúde e Enfermagem. No âmbito da educação estão incluídos estudos sobre concepções pedagógicas, formulação 
de políticas adequadas, formação de novos profissionais, e educação permanente como direito e dever profissional para fundamentar ações seguras e de qualidade.

Não existe um a priori externo a profissão, atemporal, apolítico e sem interesses econômicos e de classe, que estabeleça o que pertence e o que não pertence à Enfermagem, como bem formulado por Fourez ${ }^{(15)}$. Cada cenário é histórico, socialmente construído e resulta da luta política, portanto cabe aos próprios profissionais de Enfermagem definir parâmetros do que é requerido para uma prática adequada e segura, para profissionais e usuários ${ }^{(16)}$. Esses parâmetros tem que ser fundamentados em pesquisas, servindo de subsídio para as intervenções políticas de suas entidades organizativas. Segundo Freidson ${ }^{(5)}$ a expertise e o "credencialismo" sustentam as profissões e garantem a autonomia profissional. Neste sentido, é fundamental dispor de entidades que defendam o que cabe à profissão e que se posicionem contra o exercício ilegal. "Credencialismo" tem relação com a capacidade de seus exercentes, através de entidades representativas, convencer a sociedade e o Estado de que este trabalho é especial e necessita de condições especiais para ser exercido.

Nesta abordagem cabem algumas perguntas:

O que temos a dizer em relação ao número de profissionais de enfermagem/população? O perfil da força de trabalho da enfermagem disponível no país é adequado em quantidade e qualidade? Qual o parâmetro utilizado para definir a adequação ou não?

O que temos a dizer em relação ao custo da força de trabalho da Enfermagem? Qual a relação entre trabalho e adoecimento dos profissionais de Enfermagem? Qual a relação entre jornada de trabalho, adoecimento dos profissionais e segurança nos cuidados prestados à população?

O que temos a dizer sobre o financiamento do setor saúde? Qual a análise crítica que fazemos ao comparar com a realidade de outros países?

O que temos a dizer sobre regras requeridas para a formação profissional?

O que temos a dizer sobre os Projetos de Lei (PL) em tramitação no Congresso Nacional como o que pretende criar a "profissão de cuidadores de idosos", o PL 2295/2000 que regulamenta Jornada de Trabalho dos Profissionais de Enfermagem, e o PL conhecido como "Saúde mais 10"?

O que temos a dizer sobre políticas atuais do governo brasileiro para a saúde como: a relativa às Urgências e Emergências explicitadas em Manual Instrutivo elaborado pelo Ministério da Saúde (MS) em 2013; o Índice de Desempenho do SUS (IDSUS); o Programa Nacional de Segurança do Paciente aprovado pela Portaria MS 529 de abril de 2013; o programa "Mais Médicos"?

Todas estas iniciativas constituem-se em políticas que atendem necessidades dos usuários ou (no todo ou em parte)? De que modo as políticas prescritas interferem no trabalho da Enfermagem? E o que temos a dizer sobre pronunciamentos de autoridades governamentais que tem dado excessiva relevância a necessidade de médicos e a déficits na infraestrutura dos serviços de saúde como se saúde fosse feita com médicos, medicamentos e equipamentos médico-hospitalares?
Para avançar a ciência do cuidar é preciso fortalecer-se como profissão exercida por profissionais cidadãos que lutam para que seus exercentes possam atingir, com qualidade, a finalidade de seu trabalho. Neste sentido é prioritária uma aliança com os usuários em defesa do direito à saúde, à vida e a cuidados de qualidade, assim como é responsabilidade indelegável dos profissionais de enfermagem, definir parâmetros para a realização do seu trabalho.

\section{A ENFERMAGEM E OS DESAFIOS COMO CIÊNCIA DO CUIDAR: OS CAMINHOS DA PESQUISA}

Como "ciência do cuidar", é fundamental analisar criticamente as bases epistemológicas da produção do conhecimento disponibilizado pela profissão, no sentido de defender o rigor científico e sua utilidade social.

Cabe à profissão a reflexão filosófica sobre o que é cuidado, cuidado humano e cuidado de saúde? Qual a finalidade, a natureza e o significado do cuidado humano? O que é cuidado de Enfermagem?

Cabe produzir conhecimentos que fundamentem intervenções de Enfermagem no processo de cuidar da saúde das pessoas, da concepção à morte. Em cuidados individuais ou coletivos, no âmbito da promoção da saúde, da prevenção de doenças e da produção de tecnologias de cuidado em situações de adoecimento, de dor e sofrimento.

Articulando o "clássico e o emergente" com vistas a fortalecer a Enfermagem enquanto profissão do campo da saúde e enquanto disciplina científica do cuidado humano, cabe intervir na defesa da mudança da árvore de enquadramento das pesquisas em Enfermagem disponibilizada no site do ConseIho Nacional de Desenvolvimento Científico e Tecnológico (CNPq). O CNPq constitui-se no principal órgão financiador de pesquisadores do país e, ao apresentar determinada árvore de classificação das pesquisas, orienta o financiamento em pesquisa. Portanto, é indutor da produção de conhecimentos. Ao mesmo tempo, mostra o que é a ciência da Enfermagem. Cabe aos profissionais e pesquisadores da Enfermagem analisar, permanentemente, a sua adequação e propor mudanças. Neste processo é necessário analisar a realidade da profissão considerando o macrocenário econômico-social que define os constrangimentos institucionais e jurídico-legais no qual o seu trabalho é realizado.

$\mathrm{A}$ atual árvore de conhecimentos, para a Área da Enfermagem (Figura 1), disponível no site do $\mathrm{CNPq}^{(17)}$, é constituída por 06 sub-áreas: Enfermagem de Doenças Contagiosas, Enfermagem de Saúde Pública, Enfermagem Médico-Cirúrgica, Enfermagem Obstétrica, Enfermagem Pediátrica e Enfermagem Psiquiátrica. Este desenho é emblemático e representa a ciência normal de base positivista, além de não mostrar o que identifica a Enfermagem como disciplina da ciência e como profissão, no âmbito do trabalho coletivo em saúde.

Os pesquisadores da área de Enfermagem, os Coordenadores de Programas de Pós-Graduação e as representações da profissão na Coordenação de Aperfeiçoamento de Pessoal de Nível Superior (CAPES), no CNPq e na Associação Brasileira de Enfermagem (ABEn) têm assumido o papel requerido de 
discutir o que a Enfermagem pesquisa e o que deve pesquisar. Neste processo de debates vem sendo identificada a necessidade de mudança das subáreas disponíveis para enquadramento das pesquisas de Enfermagem no CNPq.

Figura 1- Área de Conhecimento Enfermagem e subáreas.
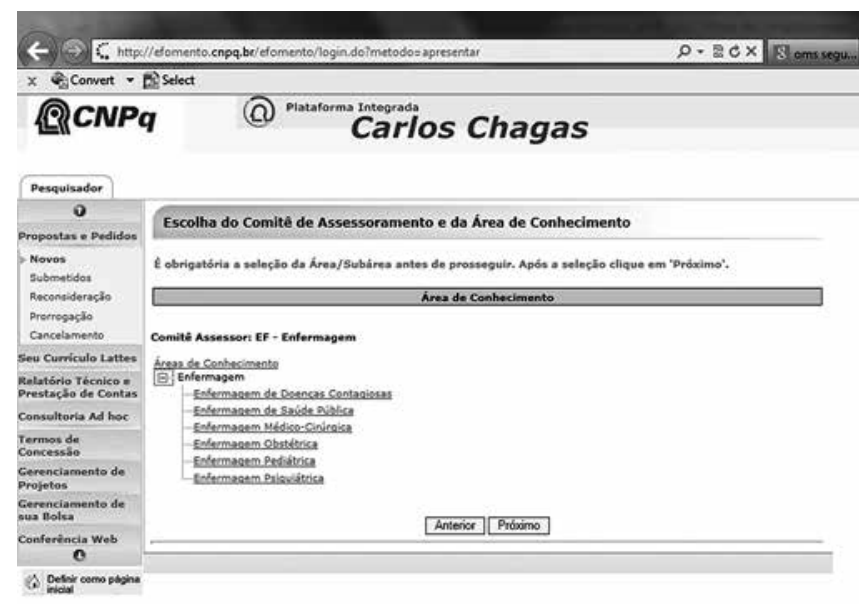

Fonte: CNPq, 2013.

Em busca de consensos provisórios (historicamente determinados, portanto provisórios) para orientar a ação, a comunidade de pesquisadores de Enfermagem elaborou uma primeira síntese coletiva em encontro ocorrido em 1998 em Niterói/ RJ, o qual reuniu a representação da área de Enfermagem na CAPES e as Coordenações de Programas de Pós-Graduação em Enfermagem do país. Essa síntese foi debatida, novamente, no ano seguinte em encontro realizado durante o SENPE, na cidade de Gramado/RS. Essa síntese coletiva organizou a produção do conhecimento em Enfermagem em três macro campos - profissional, assistencial e organizacional - sinalizando uma aproximação com o fazer e a identidade profissional e, ao mesmo tempo, a busca de um distanciamento do paradigma da biomedicina.

Em maio de 2013, as representantes da área da Enfermagem no CNPq promoveram um encontro, envolvendo pesquisadores da área, Coordenadores de Pós-Graduação e ABEn, para debater a atual configuração da árvore de enquadramento das pesquisas pelo CNPq. Neste encontro identificou-se um consenso de que o atual enquadramento não corresponde aos anseios da profissão. No entanto, não se chegou a formulação de uma nova proposta. Os diversos grupos de trabalho apresentaram propostas, sendo todas acolhidas para posterior síntese orientadora de intervenção junto a esse órgão do Ministério da Ciência e Tecnologia.

Considerando o formulado por Fourez ${ }^{(15)}$ ao tratar da filosofia da ciência, cabe explicitar que é de responsabilidade da Enfermagem definir o que lhe pertence enquanto disciplina do conhecimento científico. Considerando-se o produzido em um dos Grupos de Trabalho do referido encontro e a perspectiva de associar "o clássico e o emergente", apresento para debate uma proposta preliminar para uma "nova árvore" de enquadramento das pesquisas em Enfermagem no CNPq (Figura 2).

Figura 2 - Área e subáreas de Conhecimento em Enfermagem.

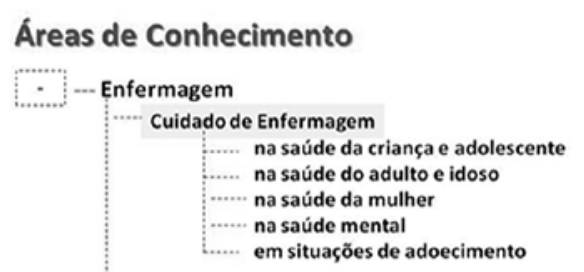

Trabalho, organização e gestão em enfermagem e saúde História, filosofia e ética em enfermagem e saúde Educação em enfermagem e saúde Tecnologias e inovação tecnológica em enfermagem e saúde Enfermagem, políticas públicas e prioridades em saúde

Fonte: Figura criada pela autora deste trabalho.

A organização se daria em 06 subáreas: Cuidado de Enfermagem; Trabalho, Organização e Gestão em Enfermagem e Saúde; História, Filosofia e Ética em Enfermagem e Saúde; Educação em Enfermagem e Saúde; Tecnologias e Inovação Tecnológica em Enfermagem e Saúde; Enfermagem, Políticas Públicas e Prioridades em Saúde.

A primeira subárea, denominada "Cuidado de Enfermagem", incluiria pesquisas que abordem práticas, ações e políticas de cuidado na dimensão individual e coletiva e desenvolvidas em todos os espaços de intervenção em saúde (públicos e privados), em todos os níveis de complexidade da assistência. Esta grande subárea seria dividida conforme as práticas de cuidado durante o ciclo vital e manteria a visibilidade de áreas já consolidadas. Ao escolher a subárea "Cuidado de Enfermagem" o pesquisador(a) escolheria, também, uma das 05 opções a serem disponibilizadas - na saúde da criança e adolescente, na saúde do adulto e idoso, na saúde da mulher, na saúde mental e em situações de adoecimento.

\section{CONSIDERAÇÕES FINAIS}

Pensar a profissão e a produção de conhecimentos requer articulação com a luta político profissional de construção de um projeto coletivo que considere intervenção no setor saúde e valorização profissional. É preciso que os profissionais de Enfermagem tenham capacidade crítica e assumam o protagonismo no setor saúde e na sociedade mostrando-se como uma profissão que defende o direito universal à saúde e a cuidados seguros e de qualidade.

Esse protagonismo só será possível com entidades e organizações representativas da profissão que atuem orientadas por preceitos éticos, fundamentadas na representação e no agir democrático, que utilizem os recursos orçamentários de forma responsável, transparente e aderente às necessidades da categoria. É necessário, também, superar a fragmentação na representação profissional, tendo como horizonte a unidade de ação, sem deixar de enfrentar as divergências e os problemas vivenciados em nossas organizações, em especial no Sistema Conselho Federal/Conselhos Regionais de Enfermagem. Atuar buscando acumular forças para conquistas que atendam profissionais e população. 


\section{REFERÊNCIAS}

1. Weber M. Economia e sociedade: fundamentos da sociologia compreensiva. Brasília: UNB; 2004.

2. Abott A. The system of professions: an essay on the division of expert labour. Chicago: The University of Chicago; 1988.

3. Moore W. The professions: roles and rules. New York: Russell Sage Foundation; 1970.

4. Wilenski HL. The professionalization of everyone? In: Grusky O, Hiller G, editores. The sociology of organizations: basic studies. New York: The Free Press; 1970.

5. Freidson E. Profissão médica: um estudo de sociologia do conhecimento aplicado. São Paulo/Porto Alegre: UNESP/ Sindicato dos Médicos; 2009.

6. Neal M, Morgan J. The professionalization of everyone? A comparative study of the development of the professions in the United Kindom and Germany. Eur Sociol Rev 2000;16(1):9-26.

7. Machado $\mathrm{MH}$, editor. Profissões de saúde: uma abordagem sociológica. Rio de Janeiro: Fiocruz; 1995.

8. Pereira FM, Pereira Neto AP. O Psicólogo no Brasil: notas sobre seu processo de profissionalização. Psicol Estud 2003;8(2):19-27.

9. Pires DEP. Hegemonia médica na saúde e a enfermagem. São Paulo: Cortez; 1989.

10. Pires DEP. Reestruturação produtiva e trabalho em saúde no Brasil. 2th ed. São Paulo: Annablume/CNTSS; 2008.
11. Pires DEP. A enfermagem enquanto disciplina, profissão e trabalho. Rev Bras Enferm 2009;62(5):739-744.

12. Bellaguarda MLR, Padilha MICSP, Pereira Neto AF, Pires DEP, Peres MAA. Reflexão sobre a legitimidade da autonomia da enfermagem no campo das profissões de saúde à luz das ideias de Eliot Freidson. Esc Anna Nery Rev Enferm 2013;17(2):369-74

13. Pires DEP, Lorenzetti J, Gelbcke FL. Enfermagem: condições de trabalho para um fazer responsável. In: Congresso Brasileiro de Enfermagem; 2010; Florianópolis, Brasil: CBEn; 2010.

14. Campos GWS. Subjetividade e administração de pessoal: considerações sobre o modo de gerenciar o trabalho em equipes de saúde. In: Merhy EE, Onocko R, editores. Agir em saúde: um desafio para o público. São Paulo: Hucitec; 1997. p. 229-266.

15. Fourez G. A construção das ciências: introdução à filosofia e à ética das ciências. São Paulo: UNESP; 1995.

16. Lorenzetti J, Pires DEP, Spricigo J, Schoeller SD. Unidade de ação: um desafio para a enfermagem brasileira. Enferm Foco 2012;3(3):152-154.

17. Conselho Nacional de Desenvolvimento Científico e Tecnológico [homepage na internet]. Escolha do Comitê de Assessoramento e Área de Conhecimento. Brasília; 2013 [acesso em 29 maio 2013]. Disponível em: <http://efomento.cnpq.br/efomento/login.do?metodo $=$ apresentar $>$. 